\title{
Heat kernels on regular graphs and generalized Ihara zeta function formulas
}

\author{
G. Chinta · J. Jorgenson · A. Karlsson
}

Received: 20 May 2014 / Accepted: 3 September 2014 / Published online: 23 September 2014 (C) Springer-Verlag Wien 2014

\begin{abstract}
We establish a new formula for the heat kernel on regular trees in terms of classical $I$-Bessel functions. Although the formula is explicit, and a proof is given through direct computation, we also provide a conceptual viewpoint using the horocyclic transform on regular trees. From periodization, we then obtain a heat kernel expression on any regular graph. From spectral theory, one has another expression for the heat kernel as an integral transform of the spectral measure. By equating these two formulas and taking a certain integral transform, we obtain as application several generalized versions of the determinant formula for the Ihara zeta function associated to finite or infinite regular graphs. Our approach to the Ihara zeta function and determinant formula through heat kernel analysis follows a similar methodology which exists for quotients of rank one symmetric spaces.
\end{abstract}

Communicated by A. Constantin.

G. Chinta and J. Jorgenson acknowledge support provided by Grants from the National Science Foundation and the Professional Staff Congress of the City University of New York. A. Karlsson received support from SNSF grant 200021_132528/1. Support from Institut Mittag-Leffler (Djursholm, Sweden) is also gratefully acknowledged by all authors.

G. Chinta · J. Jorgenson

Department of Mathematics, The City College of New York, Convent Avenue at 138th Street,

New York, NY 10031, USA

e-mail: chinta@sci.ccny.cuny.edu

J. Jorgenson

e-mail: jjorgenson@mindspring.com

A. Karlsson ( $\varangle)$

Mathematics Department, University of Geneva, 1211 Geneva, Switzerland

e-mail: anders.karlsson@unige.ch 
Keywords Heat kernels · Regular graphs - Ihara zeta function - Bessel functions

Mathematics Subject Classification Primary 05C30 - 35K08; Secondary 33A40

\section{Introduction}

Let $q$ be a positive integer and $X$ be a $(q+1)$-regular graph. There is an associated heat kernel $K_{X}\left(t, x_{0}, x\right)$ corresponding to the Laplacian formed by considering the adjacency matrix on $X$. We show that in a natural way the building blocks of $K_{X}$ are the functions

$$
q^{-r / 2} e^{-(q+1) t} I_{r}(2 \sqrt{q} t),
$$

where $r \in \mathbb{Z}_{\geq 0}$, time $t \in \mathbb{R}_{\geq 0}$, and $I_{r}$ is the classical $I$-Bessel function of order $r$. The expression for the heat kernel on $X$ comes from a new formula for the heat kernel on regular trees (Proposition 3.1) which we prove in this article. Our expression is quite different from a previous formula due to F. Chung and S.-T. Yau [7], which we describe in Subsect. 3.1, Eqs. (5a,5b). If we write the functions in the above stated building block as

$$
q^{-r / 2} \cdot e^{-(\sqrt{q}-1)^{2} t} \cdot e^{-2 \sqrt{q} t} I_{r}(2 \sqrt{q} t)
$$

then there is moreover a near-perfect analogy with the building blocks of typical heat kernel expressions on Riemannian symmetric spaces, which have the form

$$
F(r) \cdot e^{-a t} \cdot \frac{1}{\sqrt{4 \pi t} d} e^{-r^{2} / 4 t}
$$

for certain constants $a, d$ and function $F$ that allow further interpretations; we refer to the survey article [18] and the references therein for further discussion.

More precisely, we prove the following result.

Theorem 1.1 The heat kernel on a $(q+1)$-regular graph $X$ is given by

$$
K_{X}\left(t, x_{0}, x\right)=e^{-(q+1) t} \sum_{m=0}^{\infty} b_{m}(x) q^{-m / 2} I_{m}(2 \sqrt{q} t),
$$

where $I_{m}$ is the I-Bessel function of order $m, b_{m}(x)=c_{m}(x)-(q-1)\left(c_{m-2}(x)+\right.$ $\left.c_{m-4}(x)+\cdots\right)$ with the last term being either $c_{1}(x)$ or $c_{2}(x)$, and $c_{m}(x)$ is the number of geodesics from a fixed base point $x_{0}$ to $x$ of length $m \geq 0$.

To be specific, we define a geodesic in a graph to be a path without back-tracking. The terminology is consistent with concepts from Riemannian geometry where a geodesic is a path which locally is distance minimizing. Moreover, a closed geodesic is a closed path without back-tracking or tails. We defer until Sect. 2 more details on 
these definitions and a precise axiomatic characterization of the heat kernel. In view of a combinatorial observation, Proposition 2.1, we may formulate the following result.

Corollary 1.2 In addition to the stated assumptions, suppose that $X$ is vertex transitive. Let $N_{m}^{0}$ denote the number of closed geodesics of length $m$ in $X$ with base point $x_{0}$. Then

$$
K_{X}\left(t, x_{0}, x_{0}\right)=K_{q+1}\left(t, x_{0}, x_{0}\right)+e^{-(q+1) t} \sum_{m=1}^{\infty} N_{m}^{0} q^{-m / 2} I_{m}(2 \sqrt{q} t)
$$

where $K_{q+1}$ denotes the heat kernel of the $(q+1)$-regular tree.

For finite, not necessarily vertex transitive, graphs $X$, similar formulas were previously proved by Ahumada [1], Terras-Wallace [34] and Mnëv [22]. Note that our formula holds for infinite graphs as well, and is therefore more general than the finite graph case. With our methods, one can also deduce the formula for the finite nonvertex transitive case using Proposition 2.2. There is a second expression for the heat kernel coming from spectral considerations. Equating the two expressions for the heat kernel, as in known approaches to the Poisson summation formula or the Selberg trace formula, one obtains an identity which is a type of theta inversion formula. To this identity we will apply a certain integral transform, which amounts to a Laplace transform with a change of variables, and obtain the logarithmic derivative of the Ihara zeta function. This procedure is motivated by McKean [21] in his approach to the Selberg zeta function and was axiomatized in [18] to abstract settings. In the end, one obtains determinantal formulas for Ihara zeta-like functions. In a special case, we recover the standard formula stemming from Ihara's work [17], which in turn is generalized in $[2,12,15,20,33]$ for finite graphs; see Subsect. 4.3 .

We now describe one sample outcome which comes from the above described sequence of calculations. Let $X$ be a vertex transitive $(q+1)$-regular graph. We define the associated Ihara zeta function of $X$ by

$$
\zeta_{X}(u)=\exp \left\{\sum_{m=1}^{\infty} \frac{N_{m}^{0}}{m} u^{m}\right\}
$$

where $N_{m}^{0}$ is the number of closed geodesics of length $m$ starting at a fixed vertex $x_{0}$. For finite graphs, the classical Ihara zeta function is just our Ihara zeta function raised to the power equaling the number of vertices.

Theorem 1.3 Let $X$ be a vertex transitive $(q+1)$-regular graph with spectral measure $\mu$ for the Laplacian. Then

$$
\zeta_{X}(u)^{-1}=\left(1-u^{2}\right)^{(q-1) / 2} \exp \left(\int \log \left(1-(q+1-\lambda) u+q u^{2}\right) d \mu(\lambda)\right) .
$$

Again, we defer to Sect. 2 the definitions of the Laplacian and spectral measure. There are some papers in the literature defining Ihara zeta functions for infinite graphs, 
in particular Clair and Mokhtari-Sharghi [6], Grigorchuk-Zuk [14] and Guido, Isola and Lapidus [13]. Their definitions are at least a priori somewhat different in that they typically look at approximations by finite graphs and also use ideas from operator algebras such as the von Neumann trace. In many cases the different definitions coincide and the formula in Theorem 1.3 can be recovered from the above references.

A number of interesting examples of Ihara zeta functions for infinite Cayley graphs can be found in [14]. Additionally, we refer to the articles [29,30] and [31] in which the author provides a fascinating discussion in which the spectral and zeta function analysis on graphs is compared to similar studies in spectral theory on symmetric spaces and zeta functions from number theory. Going further in this direction, the results in [22] and [25] study trace formula on finite discrete graphs, and the more recent work [8] and [9] on certain cusp-like regular graphs, obtaining theorems analogous to known results in the study of the Selberg zeta function for non-compact quotients of rank one symmetric spaces.

In summary, in the present article we give a new expression for the heat kernel associated to any regular graph. One can quickly deduce the Ihara determinant formula and a number of interesting extensions, not the least of which is to infinite transitive graphs. As is well-known, one main application of such formulas is to the study of counting closed geodesics. For us, it is also significant that our analysis provides yet another instance of when the heat kernel yields zeta functions together with their main functional relation, just as in the case of Riemann, Selberg, and beyond. In particular, the present paper can be viewed in the context of the last section of [18].

\section{Preliminaries}

\subsection{Graphs}

We follow the definitions in Serre's book [28]. A graph $X$ consists of a set of vertices $V X$, a set of edges $E X$, and two maps

$$
E X \rightarrow V X \times V X, y \mapsto(o(y), t(y))
$$

and

$$
E X \rightarrow E X, y \mapsto \bar{y}
$$

such that for each $y \in E X$ we have that $\overline{\bar{y}}=y, \bar{y} \neq y$ and $o(y)=t(\bar{y})$. The vertices $o(y)$ and $t(y)$ are the extremities of the edge $y$. Two vertices are adjacent if they are extremities of an edge. The degree of a vertex $x$ is

$$
\operatorname{deg} x=\operatorname{Card}\{y \in E X: o(y)=x\}
$$

A graph is $d$-regular if each vertex has degree $d$.

There is an obvious notion of morphism. Let $P A T H_{n}$ denote the graph with vertices $0,1,2, \ldots, n$ and (half of) the edges are given by $[i, i+1], i=0, \ldots, n-1$. A 
path (of length $n$ ) is a morphism $c$ from $P A T H_{n}$ into the graph. The sequences of edges $y_{i}=c([i, i+1])$ [such that $\left.t\left(y_{i}\right)=o\left(y_{i+1}\right)\right]$ determines the path. In particular a path is oriented. There is a backtracking if for some $i$ that $y_{i+1}=\overline{y_{i}}$ and there is a tail if $y_{0}=\bar{y}_{n-1}$. A path is closed if $c(0)=c(n)$. A geodesic is a path without backtracking. A geodesic loop (or circuit in Serre's terminology) is a closed path that is a geodesic. A closed geodesic is a closed path with no tail and without backtracking.

Let us here make a comment on the terminology we have chosen. In graph theory the names for what we call closed geodesics or geodesic loops, range from circuits, loops etc, to closed paths without backtracking and no tails. Our terminology is instead directly imported from Riemannian geometry for the fully analogous concepts. In that theory geodesics are locally distance minimizing paths and the difference between a geodesic loop and a closed geodesic is that the latter is required to be differentiable also at the starting/ending point.

The path of length zero counts as a closed geodesic and, therefore, is a geodesic loop. Additionally, every closed path with one edge counts as a closed geodesic. Any length two geodesic loop is also a closed geodesic, but the closed path $y \cdot \bar{y}$ is neither.

A prime geodesic is an equivalence class of closed geodesics $[c]$ (where the equivalence class is forgetting the starting point) which is primitive in the sense that it not a power of another closed geodesic. The latter means by definition that there is no closed geodesic $d$ and integer $n>1$ such that $[c]=\left[d^{n}\right]$, which says in words that $c$ is not just a geodesic that traverses another one $n$ number of times.

An orientation is a subset $E X_{+}$of edges such that $E X$ is the disjoint union of $E X_{+}$and $\overline{E X_{+}}$. With the data of a graph one can associate a geometric realization: start with the discrete topology, take $V X \times[0,1]$ and make identification based on the maps $o$ and $t$.

A tree is a connected nonempty graph without geodesic loops.

We will in particular consider vertex transitive graphs. This means there is a group of automorphisms which is transitive on the vertices. In particular such a graph is of course regular. A rich source of such graphs is provided by Cayley graphs of groups: Let $G$ be a group and let $S$ be a subset of $G$. We denote by $X(G, S)$ the oriented graph having elements of $G$ as vertices and $E X_{+}=G \times S$ with $o(g, s)=g$ and $t(g, s)=g s$ for each edge $(g, s)$.

Let $X$ be a graph on which a group $G$ acts. An inversion is a pair consisting of an element $g$ and an edge $y$ such that $g y=\bar{y}$. If $G$ acts without inversions (which is the same as saying that there is an orientation of $X$ preserved by $G$ ) we can define the quotient graph $G \backslash X$ in an obvious way; the respective edge and vertex sets are the corresponding quotients. (To get rid of inversions one may pass to a barycentric division.) As in topology we say that $X$ is a regular covering of $Y$ if there is a group which acts on $X$ freely and without inversion with quotient $Y$.

Let $X$ be a $(q+1)$-regular graph. Then its universal covering is the $(q+1)$-regular tree, and the covering group acts freely on the tree without inversion. The covering group is a free group. 


\subsection{Path counting in graphs}

We fix a base vertex $x_{0}$ in a graph $X$ and define the following counting functions which will be used in this paper:

- $a_{k}(x)$ is the number of paths of length $k$ from $x_{0}$ to $x$,

- $c_{k}(x)$ is the number of geodesics of length $k$ from $x_{0}$ to $x$,

- $c_{k}^{0}=c_{k}\left(x_{0}\right)$ is the number of geodesic loops of length $k$ starting at $x_{0}$,

- $c_{k}$ the number of geodesic loops of length $k$, from some starting point with a distinct direction,

- $N_{k}^{0}$ is the number of closed geodesics of length $k$ starting at $x_{0}$,

- $N_{k}$ is the number of closed geodesics of length $k$, from some starting point with a distinct direction,

- $\pi_{k}$ is the number of prime geodesics of length $k$.

The sequences $\left\{c_{k}\right\},\left\{N_{k}\right\}$ and $\left\{\pi_{k}\right\}$ only make sense for finite graphs, since if the graph is infinite, these values are typically infinite. For finite vertex transitive graphs, the sequences $\left\{N_{k}^{0}\right\}$ and $\left\{N_{k}\right\}$ are related by the number of vertices, i.e. starting points. Specifically, if the graph $X$ has $n$ vertices, then $N_{k}^{0} \cdot n=N_{k}$ for all $k$. Also, $N_{k}$ and $\pi_{k}$ have a precise relationship, see e.g. [32] or [14]. Finally, we recall the conventions that $c_{0}^{0}=N_{0}^{0}=1, c_{1}^{0}=N_{1}^{0}$ and $c_{2}^{0}=N_{2}^{0}$.

Example Let $X$ be the complete graph on four vertices. To illustrate and clarify the definitions above we note that $c_{0}^{0}=1, c_{0}^{1}=0, c_{2}^{0}=0, c_{3}^{0}=6, c_{4}^{0}=12$, and $c_{5}^{0}=12$. The values for $N_{k}^{0}$ coincides with $c_{k}^{0}$ until $k=4$ but then $N_{5}^{0}=0$.

Proposition 2.1 Let $X$ be a transitive $(q+1)$-regular graph. Then for $k \geq 3$, following relation holds true

$$
N_{k}^{0}=c_{k}^{0}-(q-1)\left(c_{k-2}^{0}+c_{k-4}^{0}+\cdots\right)
$$

the last term being $c_{1}^{0}$ or $c_{2}^{0}$ depending on the parity of $k$.

Proof This is similar to an argument in [27]. A geodesic loop of length $k \geq 3$ which is not a closed geodesic has the form $y_{1} \cdot z \cdot \bar{y}_{1}$ where $z$ is a geodesic loop of length $k-2$. There are two possibilities, either $z$ is a closed geodesic or not. If we fix $z$, then the number of possibilities for $y_{1}$ is $q-1$ in the first case and $q$ in the second case. Since $X$ is vertex transitive, we may freely change the starting point of any loop, namely $z$. With this in mind, we obtain the recursive relation that

$$
c_{k}^{0}-N_{k}^{0}=(q-1) N_{k-2}^{0}+q\left(c_{k-2}^{0}-N_{k-2}^{0}\right)=\left(c_{k-2}^{0}-N_{k-2}^{0}\right)+(q-1) c_{k-2}^{0},
$$

which we can write as

$$
N_{k}^{0}-N_{k-2}^{0}=c_{k}^{0}-q c_{k-2}^{0}
$$

Using that $c_{1}^{0}=N_{1}^{0}$ and $c_{2}^{0}=N_{2}^{0}$, the proposition follows by induction on $k$. 
With a proof similar to the one given in the above proposition, we obtain the following result.

Proposition 2.2 Let $X$ be a finite $(q+1)$-regular graph. Then for $k \geq 3$, the following relation holds true

$$
N_{k}=c_{k}-(q-1)\left(c_{k-2}+c_{k-4}+\cdots\right)
$$

the last term being $c_{1}$ or $c_{2}$ depending on the parity of $k$.

\subsection{The combinatorial laplacian and heat kernel}

Given a $(q+1)$-regular graph $X$ and function $f$ on the vertices $X$, the Laplacian of $f$, written as $\Delta f$, is the function of the vertices of $X$ which is defined by the formula

$$
\Delta f(x)=(q+1) f(x)-\sum_{e \text { s.t. } o(e)=x} f(t(e)) .
$$

The Laplacian is a semi-positive, bounded self-adjoint operator on $L^{2}(V X)$. For a finite graph with $N$ vertices we label the eigenvalues of $\Delta$ as follows: $0=\lambda_{0} \leq \lambda_{1} \leq$ $\cdots \leq \lambda_{N-1} \leq 2(q+1)$. The heat kernel $K_{X}(t, x, y): \mathbb{R}_{\geq 0} \times X \times X \rightarrow \mathbb{R}$ on $X$ is the solution of

$$
\begin{aligned}
\Delta K_{X}\left(t, x_{0}, x\right)+\frac{\partial}{\partial t} K_{X}\left(t, x_{0}, x\right) & =0 \\
K_{X}\left(0, x_{0}, x\right) & = \begin{cases}1 & \text { if } x=x_{0} \\
0 & \text { otherwise }\end{cases}
\end{aligned}
$$

Sometimes we write $K_{X}\left(t, x_{0}, x\right)=K_{X}(t, x)$ when a base point $x_{0}$ is understood.

Whenever $X$ is a countable graph with bounded vertex degree, the heat kernel of $X$ exists and is unique among bounded functions $([10,11])$. Let $X$ be a regular covering of $Y$ via the map $\pi$ and group $\Gamma$, then it is formally immediate that

$$
K_{Y}\left(t, y_{0}, y\right)=\sum_{x \in \pi^{-1}(y)} K_{X}\left(t, x_{0}, x\right)
$$

where $y_{0}=\pi\left(x_{0}\right)$; alternatively, with a chosen $x$ such that $y=\pi(x)$, we have that

$$
K_{Y}\left(t, y_{0}, y\right)=\sum_{\gamma \in \Gamma} K_{X}\left(t, x_{0}, \gamma x\right) \text {. }
$$

One can show that the heat kernel decays sufficiently rapidly so that the above equalities are not only formal, but indeed are convergent series. We refer to $[4,10]$ as well as the heat kernel formula for trees in Proposition 3.1 and the bounds in Subsect. 2.5. 
Consider the numbers $a_{n}(x)$ defined by

$$
e^{(q+1) t} K_{X}(t, x)=\sum_{n=0}^{\infty} a_{n}(x) \frac{t^{n}}{n !}
$$

then it is well-known and simple to see that $a_{n}(x)$ is the number of paths from $x_{0}$ to $x$ as defined above.

\subsection{The $I$-Bessel function}

Classically, the $I$-Bessel function $I_{x}(t)$ is defined as a certain solution to the differential equation

$$
t^{2} \frac{d^{2} w}{d t^{2}}+t \frac{d w}{d t}-\left(t^{2}+x^{2}\right)=0 .
$$

For integer values of $x$, it is immediately shown that $I_{x}=I_{-x}$ and, for positive integer values of $x$, we have the series representation

$$
I_{x}(t)=\sum_{n=0}^{\infty} \frac{(t / 2)^{2 n+x}}{n ! \Gamma(n+1+x)}
$$

as well as the integral representation

$$
I_{x}(t)=\frac{1}{\pi} \int_{0}^{\pi} e^{t \cos (\theta)} \cos (\theta x) d \theta .
$$

The mathematical literature contains a vast number of articles and monographs which study the many fascinating properties and manifestations of the $I$-Bessel functions, as well as other Bessel functions. The connection with the discrete heat equation comes from the basic relation

$$
I_{x+1}(t)+I_{x-1}(t)=2 \frac{d}{d t} I_{x}(t),
$$

which easily can be derived from the integral representation and trigonometric identities. This relation will be used in the proof of Proposition 3.1.

\subsection{Universal bounds for the $I$-Bessel function}

We have the following uniform bounds from [4] which used [26]. For any $t>0$ and integer $x \geq 0$, we have that

$$
\sqrt{t} \cdot e^{-t} I_{x}(t) \leq\left(\frac{t}{t+x}\right)^{x / 2}=\left(1+\frac{x}{t}\right)^{-x / 2}
$$


As stated, the above bound is enough to show that the periodization procedure in our setting gives rise to convergent sum expressions for the heat kernel.

\subsection{An integral transform of I-Bessel}

For integers $n$ and $s \in \mathbf{C}$ with $\operatorname{Re}(s)>0$ we have e.g. from [24], that

$$
\int_{0}^{\infty} e^{-s t} e^{-t} I_{n}(t) d t=\frac{\left(s+1-\sqrt{\left(s^{2}+2 s\right)}\right)^{n}}{\sqrt{\left(s^{2}+2 s\right)}} .
$$

We will consider the transform, essentially the Laplace transform,

$$
G f(u)=\left(u^{-2}-q\right) \int_{0}^{\infty} e^{-(q u+1 / u) t} e^{(q+1) t} f(t) d t .
$$

In view of the above formula, applying the transform to the heat kernel building block, we get

$$
G\left(e^{-(q+1) t} q^{-k / 2} I_{k}(2 \sqrt{q} t)\right)(u)=u^{k-1}
$$

for $k \geq 0$ and $0<u<1 / \sqrt{q}$.

\section{Heat kernels on regular graphs}

\subsection{A heat kernel expression for regular trees}

Let $X$ be the $(q+1)$-regular tree and $x_{0} \in X$ a base point. From its characterizing properties (1a) and (1b), it is immediate to show that the heat kernel on a graph is invariant with respect to any graph automorphism $g$ :

$$
K\left(t, g x_{0}, g x\right)=K\left(t, x_{0}, x\right) .
$$

In particular, we have that the heat kernel $K\left(t, x_{0}, x\right)$ on the tree $X$ is radial, that is it depends only on $r=d\left(x_{0}, x\right)$. Therefore we can write the heat kernel as $K(t, r)$. Expressions for $K(t, r)$ were established by Bednarchak [3], Chung and Yau [7], Cowling, Meda and Setti [5], as well as Horton, Newland and Terras [16]. In the physics literature regular trees are called Bethe lattices. As stated, one of the main results of this paper is a formula for the heat kernel on $X$, which we consider to be new since we did not find the expression in either the mathematical or physics literature.

As an example of a known expression, Chung and Yau [7] prove that in the radial coordinate $r$, the heat kernel of the $(q+1)$-regular tree is given by 


$$
K(t, r)=\frac{2 e^{-(q+1) t}}{\pi q^{r / 2-1}} \int_{0}^{\pi} \frac{\exp (2 t \sqrt{q} \cos u) \sin u(q \sin (r+1) u-\sin (r-1) u)}{(q+1)^{2}-4 q \cos ^{2} u} d u
$$

for $r>0$, and

$$
K(t, 0)=\frac{2 q(q+1) e^{-(q+1) t}}{\pi} \int_{0}^{\pi} \frac{\exp (2 t \sqrt{q} \cos u) \sin ^{2} u}{(q+1)^{2}-4 q \cos ^{2} u} d u .
$$

The formula which we prove is given in the following proposition.

Proposition 3.1 The heat kernel of the $(q+1)$-regular tree is given in the radial coordinate $r \geq 0$ as

$$
K(t, r)=q^{-r / 2} e^{-(q+1) t} I_{r}(2 \sqrt{q} t)-(q-1) \sum_{j=1}^{\infty} q^{-(r+2 j) / 2} e^{-(q+1) t} I_{r+2 j}(2 \sqrt{q} t),
$$

where I denotes the I-Bessel function.

Proof It is immediate that when $t=0$, the above series is equal to $\delta_{0}(r)$ as required, since $I_{x}(0)=0$ for $x \neq 0$ and $I_{0}(0)=1$. Since $K(0, r)=\delta_{0}(r)$, it remains to show that the above series is equal to $K(t, 0)$ for $t>0$. Denote by $f(r)=K(t, r)$ and $\dot{f}(r)=\partial K(t, r) / \partial t$. If $r=0$, then the differential equation (1a) for the heat kernel takes the form

$$
(q+1) f(0)-(q+1) f(1)+\dot{f}(0)=0,
$$

and for $r>0$ the differential equation becomes

$$
(q+1) f(r)-q f(r+1)-f(r-1)+\dot{f}(r)=0 .
$$

Let $g(r)$ be the above series expansion times $e^{(q+1) t}$, or, when written out,

$$
g(r)=q^{-r / 2} I_{r}(2 \sqrt{q} t)-(q-1) \sum_{j=1}^{\infty} q^{-(r+2 j) / 2} I_{r+2 j}(2 \sqrt{q} t)
$$

It is an elementary exercise to show that the series expansion satisfies the characterizing differential equation for the heat kernel if and only if we have the differential equations

$$
-(q+1) g(1)+\dot{g}(0)=0,
$$

and for every $r>0$

$$
-q g(r+1)-g(r-1)+\dot{g}(r)=0 .
$$


Let us first verify (6). We begin by writing the

$$
\begin{aligned}
\text { Left-hand-side of }(6)= & -(q+1) q^{-1 / 2} I_{1}(2 \sqrt{q} t) \\
& +(q+1)(q-1) \sum_{j=1}^{\infty} q^{-(1+2 j) / 2} I_{1+2 j}(2 \sqrt{q} t) \\
& +2 \sqrt{q} I_{0}^{\prime}(2 \sqrt{q} t)-(q-1) 2 \sqrt{q} \sum_{j=1}^{\infty} q^{-2 j} I_{2 j}^{\prime}(2 \sqrt{q} t) .
\end{aligned}
$$

Using the basic relation $I_{r-1}(z)+I_{r+1}(z)=2 I_{r}^{\prime}(z)(4)$, we obtain the expression

$$
\begin{aligned}
\text { Left-hand-side of }(6)= & -(q+1) q^{-1 / 2} I_{1}(2 \sqrt{q} t) \\
& +(q+1)(q-1) \sum_{j=1}^{\infty} q^{-(1+2 j) / 2} I_{1+2 j}(2 \sqrt{q} t) \\
& +\sqrt{q}\left(I_{1}(2 \sqrt{q} t)+I_{-1}(2 \sqrt{q} t)\right) \\
& -(q-1) \sqrt{q} \sum_{j=1}^{\infty} q^{-j}\left(I_{2 j+1}(2 \sqrt{q} t)+I_{2 j-1}(2 \sqrt{q} t)\right) .
\end{aligned}
$$

Collecting terms, and recalling that $I_{-1}=I_{1}$, we can evaluate the coefficient of each $I$-Bessel function in (8):

$$
\begin{aligned}
I_{0} & : \text { there are no } I_{0} \text { terms, } \\
I_{1} & :-(q+1) q^{-1 / 2}+2 \sqrt{q}-(q-1) \sqrt{q} q^{-1}=0, \\
I_{2 j+1} & :\left(q^{2}-1\right) q^{-(1+2 j) / 2}-(q-1) \sqrt{q} q^{-j}-(q-1) \sqrt{q} q^{-(j+1)} \\
& =(q-1) q^{-j}\left((q+1) q^{-1 / 2}-q^{1 / 2}-q^{-1 / 2}\right) \\
& =0 .
\end{aligned}
$$

In other words, the left-hand-side of (6) is zero, as required.

Let us now check the case when $r>0$. Again, we begin by writing the

$$
\begin{aligned}
\text { Left-hand-side of }(7)= & -q^{1-(r+1) / 2} I_{r+1}(2 \sqrt{q} t) \\
& +q(q-1) \sum_{j=1}^{\infty} q^{-(r+1+2 j) / 2} I_{r+1+2 j}(2 \sqrt{q} t) \\
& -q^{-(r-1) / 2} I_{r-1}(2 \sqrt{q} t) \\
& +(q-1) \sum_{j=1}^{\infty} q^{-(r-1+2 j) / 2} I_{r-1+2 j}(2 \sqrt{q} t)
\end{aligned}
$$




$$
\begin{aligned}
& +2 \sqrt{q} q^{-r / 2} I_{r}^{\prime}(2 \sqrt{q} t) \\
& -2 \sqrt{q}(q-1) \sum_{j=1}^{\infty} q^{-(r+2 j) / 2} I_{r+2 j}^{\prime}(2 \sqrt{q} t) \\
& =-q^{1 / 2-r / 2} I_{r+1}(2 \sqrt{q} t) \\
& +q(q-1) \sum_{j=1}^{\infty} q^{-(r+1+2 j) / 2} I_{r+1+2 j}(2 \sqrt{q} t) \\
& -q^{-(r-1) / 2} I_{r-1}(2 \sqrt{q} t) \\
& +(q-1) \sum_{j=1}^{\infty} q^{-(r-1+2 j) / 2} I_{r-1+2 j}(2 \sqrt{q} t) \\
& +\sqrt{q} q^{-r / 2}\left(I_{r+1}(2 \sqrt{q} t)\right. \\
& \left.+I_{r-1}(2 \sqrt{q} t)\right) \\
& +\sqrt{q}(q-1) \sum_{j=1}^{\infty} q^{-(r+2 j) / 2}\left(I_{r+1+2 j}(2 \sqrt{q} t)\right. \\
& \left.+I_{r-1+2 j}(2 \sqrt{q} t)\right) .
\end{aligned}
$$

As above, we can evaluate the coefficient of each $I$-Bessel function in (9):

$$
\begin{aligned}
I_{r-1}: & -q^{-(r-1) / 2}+\sqrt{q} q^{-r / 2}=0 \\
I_{r}: & \text { there are no } I_{r} \text { terms } \\
I_{r+1}: & -q^{1 / 2-r / 2}+(q-1) q^{-(r+1) / 2}+\sqrt{q} q^{-r / 2}-\sqrt{q}(q-1) q^{-(r+2) / 2}=0 \\
I_{r+2 j+1}: & q(q-1) q^{-(r+1+2 j) / 2}+(q-1) q^{-(r+1+2 j) / 2} \\
& -\sqrt{q}(q-1) q^{-(r+2 j) / 2}-\sqrt{q}(q-1) q^{-(r+2 j+2) / 2} \\
= & (q-1) q^{-r / 2} q^{-j}\left(q^{1 / 2}+q^{-1 / 2}-q^{1 / 2}-q^{-1 / 2}\right) \\
= & 0 .
\end{aligned}
$$

In other words, the left-hand-side of (7) is zero, as required, which completes the proof of the proposition.

In the following subsection we indicate another approach to the proof of Proposition 3.1.

\subsection{The horospherical transform}

Every geodesic ray $\gamma$ in the tree emanating from a fixed base point $x_{0}$ can be viewed as an "ideal boundary point at infinity". To each such $\gamma$ there are associated horospheres, one for each integer $n$ : 


$$
\mathcal{H}_{n}=\left\{x \in X: \lim _{k \rightarrow \infty}[d(\gamma(k), x)-k]=n\right\}
$$

where $d$ is the natural combinatorial distance in the graph.

We fix a geodesic ray $\gamma$ and may then consider the associated horospherical transform of functions $f: X \rightarrow \mathbb{R}$ denoted by

$$
\mathbf{H} f: \mathbb{Z} \rightarrow \mathbb{R}
$$

and defined by $\mathbf{H} f(n)=\sum_{x \in \mathcal{H}_{n}} f(x)$. For a radial function, decaying fast enough, we have the inversion formula

$$
f(r)=q^{-r}(\mathbf{H} f)(r)-(q-1) \sum_{j=1}^{\infty} q^{-(r+2 j)}(\mathbf{H} f)(r+2 j)
$$

for $r \geq 0$. This is stated in [16] on pages 7-8 for $f$ of finite support.

If we apply the horospherical transform to the Eqs. (1a, 1b) characterizing the heat kernel, we get

$$
(q+1) \mathbf{H} K(t, n)-(q \mathbf{H} K(t, n+1)+\mathbf{H} K(t, n-1))+\frac{\partial}{\partial t} \mathbf{H} K(t, n)=0,
$$

for $n \in \mathbb{Z}$ and with $\mathbf{H} K(0, n)=\delta_{0}(n)$. The solution to this difference-differential equation can be seen to be (cf. section 3.2 in [16] or [19])

$$
f(t, n)=q^{-n / 2} e^{-(q+1) t} I_{n}(2 \sqrt{q} t) .
$$

As already remarked, the heat kernel on a regular tree is radial, so by inserting the above expression into the inversion formula (11) (here we need to go beyond finitely supported functions for which this formula was stated in [16]) we can get a different proof of Proposition 3.1.

\subsection{Heat kernels on regular graphs}

Let $q>0$ be an integer and $X$ a $(q+1)$-regular graph. We fix a base point $x_{0} \in V X$ which we will suppress in the notation. The heat kernel on $X$ can be obtained from periodizing the heat kernel $K_{q+1}$ on the universal covering space, the $(q+1)$-regular tree $T_{q+1}$, over the covering group $\Gamma$ Following the remarks in Subsect. 2.3 we have (with a slight abuse of notation) that

$$
K_{X}(t, x)=\sum_{\gamma \in \Gamma} K_{q+1}(t, \gamma x)
$$

Recall that $c_{n}(x)$ denotes the number of paths without backtracking from the identity $x_{0}$ to $x$ of length $n$ in $X$. We also use $c_{n}^{0}=c_{n}\left(x_{0}\right)$ as notation for the number of geodesic loops, i.e. closed paths without backtracking, starting at $x_{0}$. Note that $c_{n}(x)$ 
is equal to the number of elements of the form $\gamma x$ for some $\gamma \in \Gamma$ on the radius $n$ sphere in $T_{q+1}$. We therefore have

$$
K_{X}(t, x)=\sum_{n \geq 0} c_{n}(x) K_{q+1}(t, n)
$$

or more explicitly by inserting the expression from Proposition 3.1 for $K_{q+1}(t, n)$,

$$
K_{X}(t, x)=e^{-(q+1)} \sum_{n \geq 0} c_{n}(x) \sum_{j=0}^{\infty} d_{q}(j) q^{-n / 2-j} I_{n+2 j}(2 \sqrt{q} t)
$$

where $d_{q}(j)$ is 1 if $j=0$ and $1-q$ otherwise. A rearrangement of the terms gives

$$
K_{X}(t, x)=e^{-(q+1) t} \sum_{m \geq 0} b_{m}(x) q^{-m / 2} I_{m}(2 \sqrt{q} t),
$$

where $b_{m}(x)=c_{m}(x)-(q-1)\left(c_{m-2}(x)+c_{m-4}(x)+\cdots\right)$ where the last term is $c_{1}(x)$ if $m$ is odd and $c_{0}(x)$ if $m$ is even. This is also with the understanding that $b_{0}(x)=c_{0}(x)$ and $b_{1}(x)=c_{1}(x)$. This proves Theorem 1.1.

Now specialize to $x=x_{0}$. In view of Propositions 2.1 and 3.1 we obtain Corollary 1.2 as well.

\subsection{Spectral theory}

An excellent reference here is that of Mohar and Woess [23]. One has that there are spectral measures $\mu_{x}$ such that (suppressing $x_{0}$ )

$$
K_{X}(t, x)=\int e^{-\lambda t} d \mu_{x}(\lambda)
$$

In particular if $X$ is a finite graph with $n$ vertices, then the Laplacian has eigenvalues $0=\lambda_{0}<\lambda_{1} \leq \cdots \leq \lambda_{n-1}$ and corresponding orthonormal eigenfunctions $\phi_{j}$. The heat kernel may thus be written as

$$
K_{X}\left(t, x_{0}, x\right)=\frac{1}{n} \sum_{j=0}^{n-1} e^{-\lambda_{j} t} \phi_{j}(x) \overline{\phi_{j}\left(x_{0}\right)}
$$

\section{Ihara formulas}

In this final section we describe how the $G$-transform introduced in Sect. 2.6 applied to the heat kernel gives rise to the Ihara zeta function. 


\subsection{Zeta functions}

Motivated by Selberg's work, Ihara defined a zeta function for a finite graph $X$, which is now referred to as the Ihara zeta function. The product formula for the Ihara zeta function is

$$
\zeta_{X}^{I h}(u)=\prod_{[P]}\left(1-u^{l(P)}\right)^{-1}
$$

where the product is over equivalence classes of prime geodesics and $l$ the length. (Actually, Ihara worked in a specific group setting, but Serre remarked in the preface of [28] that the definition could be given a simple interpretation in terms of graphs.) By a general calculation, see for example [32, p. 29], one has

$$
\log \zeta_{X}^{I h}(u)=\sum_{m=1}^{\infty} \frac{N_{m}}{m} u^{m}
$$

where $N_{m}$ is the number of closed geodesics of length $m$. Thus the Ihara function is a zeta type function similar to those appearing in the classical works of Artin, Hasse, and Weil on counting points of varieties in finite fields.

The numbers $N_{m}$ are not defined for infinite graphs $X$. In the case of transitive graphs, a natural replacement is $N_{m}^{0}$, since in the finite case one has $N_{m}=n N_{m}^{0}$, where $n$ is the number of vertices. So we can define a zeta function for any (not necessarily finite) vertex transitive graph via

$$
\log \zeta_{X}(u)=\sum_{m=1}^{\infty} \frac{N_{m}^{0}}{m} u^{m}
$$

More exotically one could define a two variable function $\zeta$ via

$$
\log \zeta_{X}(u, x)=\sum_{m=1}^{\infty} \frac{b_{m}(x)}{m} u^{m}
$$

Following Riemann one has another set of zeta functions by instead taking the Mellin transform of the the heat kernel. This is a subject for another paper.

\subsection{The periodization side}

We will apply the transform

$$
G f(u)=\left(u^{-2}-q\right) \int_{0}^{\infty} e^{-(q u+1 / u) t} e^{(q+1) t} f(t) d t,
$$

where we throughout consider $0<u<1 / \sqrt{q}$. First we transform our heat kernel expression 


$$
K_{X}\left(t, x_{0}, x\right)=e^{-(q+1) t} \sum_{m=0}^{\infty} b_{m}(x) q^{-m / 2} I_{m}(2 \sqrt{q} t)
$$

of Theorem 1.1. Using the basic formula in Subsect. 2.6, in the case $x \neq x_{0}$, so $b_{0}(x)=0$ we have that the $G$-transform of the heat kernel is equal to

$$
\frac{1}{u} \sum_{m=0}^{\infty} b_{m}(x) u^{m}=\frac{\partial}{\partial u} \sum_{m=1}^{\infty} \frac{b_{m}(x)}{m} u^{m}=\frac{\partial}{\partial u} \log \zeta_{X}(u, x) .
$$

In the case $x=x_{0}$, the $G$-transform of the heat kernel on the diagonal is equal to

$$
\frac{1}{u} \sum_{m=0}^{\infty} b_{m} u^{m}=\frac{\partial}{\partial u} \sum_{m=1}^{\infty} \frac{b_{m}}{m} u^{m}+\frac{\partial}{\partial u} \log u
$$

In the vertex transitive case, for the case $x=x_{0}$ we apply the transform to the expression of Corollary 1.2

$$
K_{X}\left(t, x_{0}, x_{0}\right)=K_{q+1}\left(t, x_{0}\right)+e^{-(q+1) t} \sum_{m=1}^{\infty} N_{m}^{0} q^{-m / 2} I_{m}(2 \sqrt{q} t)
$$

which gives (summing the geometric series arising from the first term)

$$
1 / u-(q-1) \frac{u}{1-u^{2}}+\frac{1}{u} \sum_{m=1}^{\infty} N_{m}^{0} u^{m}
$$

This can in turn be written as

$$
\begin{aligned}
& \frac{\partial}{\partial u} \log u+\frac{q-1}{2} \frac{\partial}{\partial u} \log \left(1-u^{2}\right)+\frac{\partial}{\partial u} \sum_{m=1}^{\infty} \frac{N_{m}^{0}}{m} u^{m} \\
& =\frac{\partial}{\partial u} \log u+\frac{q-1}{2} \frac{\partial}{\partial u} \log \left(1-u^{2}\right)+\frac{\partial}{\partial u} \log \left(\zeta_{X}(u)\right) .
\end{aligned}
$$

We have proven

Proposition 4.1 For X a $(q+1)$-regular vertex transitive graph,

$$
\left(G K_{X}\right)\left(\cdot, x_{0}\right)(u)=\frac{\partial}{\partial u}\left[\log u+\frac{q-1}{2} \log \left(1-u^{2}\right)+\log \zeta_{X}(u) .\right]
$$

In summary, the $G$-transform of the heat kernel yields expressions involving the Ihara zeta function together with other trivial terms. In the setting of compact quotients of rank one symmetric spaces, there is a similar change of variables in the Laplace transform so that when applied to the trace of the heat kernel, one obtains the Selberg zeta function. With this in mind, our approach to the Ihara zeta function as an integral 
transform of the heat kernel is in line with a known approach to the Selberg zeta function in many settings.

\subsection{Ihara's determinantal formula}

Now we deduce the classical Ihara determinantal formula. Let $X$ be a $(q+1)$-regular graph of finite vertex cardinality $n$. From (13),

$$
K_{X}\left(t, x_{0}\right)=\frac{1}{n} \sum_{j=0}^{n-1} e^{-\lambda_{j} t}
$$

The $G$-transform of the righthand side is a simple integration which yields

$$
\begin{aligned}
& \frac{1}{n}\left(u^{-2}-q\right) \sum_{j=0}^{n-1} \frac{1}{q u+1 / u-\left(q+1-\lambda_{j}\right)} \\
& \quad=-\frac{1}{n} \frac{\partial}{\partial u} \sum_{j=0}^{n-1} \log \frac{1}{u}\left(1-\left(q+1-\lambda_{j}\right) u+q u^{2}\right) .
\end{aligned}
$$

Comparing this last expression with Proposition 4.1 from the periodization side (and also verifying that integration constants match up) we immediately get Ihara's formula, namely

$$
\frac{1}{\zeta_{X}^{I h}(u)}=\left(1-u^{2}\right)^{n(q-1) / 2} \operatorname{det}\left(\left(1-(q+1) u+q u^{2}\right) I+\Delta u\right),
$$

since, as remarked above,

$$
\frac{1}{u} \sum_{m=1}^{\infty} N_{m} u^{m}=\frac{\partial}{\partial u} \log \zeta_{X}^{I h}(u)
$$

This formula is also known to hold more generally for non-regular graphs, see the references mentioned in the introduction.

\subsection{First extension of Ihara's formula}

Using spectral theory, we obtain a similar formula for infinite transitive graphs, this time using our zeta function instead of Ihara's. The common point is that both zeta functions are obtained as $G$-transforms of the heat kernel, and the determinantal formula follows from having another expression for the heat kernel, namely that which comes from spectral theory.

We use the notation $\mu=\mu_{x_{0}}$. Since the functions involved are positive, we may change the order of integration in our integral transforms and arrive at the expres- 
sion Equating the expression in Proposition 4.1 with the $G$-transform of the spectral expansion of the heat kernel given in (12), we arrive at

$$
\begin{aligned}
& \frac{\partial}{\partial u}\left[\log u+\frac{q-1}{2} \log \left(1-u^{2}\right)+\log \zeta_{X}(u)\right] \\
& =-\frac{\partial}{\partial u} \int \log \frac{1}{u}\left(1-(q+1-\lambda) u+q u^{2}\right) d \mu(\lambda)
\end{aligned}
$$

Note: since the functions involved are positive, we are justified in interchanging the spectral integral with the $G$-transform integral on the righthand side. We now integrate this equality, noting that at $u=0$ both sides are 0 to determine the integration constants. We get the formula

$\log u+\frac{q-1}{2} \log \left(1-u^{2}\right)+\log \zeta_{X}(u)=-\int \log \frac{1}{u}\left(1-(q+1-\lambda) u+q u^{2}\right) d \mu(\lambda)$,

which leads to

$$
\zeta_{X}(u)^{-1}=\left(1-u^{2}\right)^{(q-1) / 2} \exp \left[\int \log \left(1-(q+1-\lambda) u+q u^{2}\right) d \mu(\lambda)\right] .
$$

This is Theorem 1.3. We further remark that Eq. (14) clearly generalizes the Ihara determinant formula since for vertex transitive graphs with a finite number $n$ vertices one has $\zeta_{X}^{I h}=\zeta_{X}^{n}$.

\subsection{Second extension of Ihara's formula}

Here we do not specialize to $x=x_{0}$. The resulting identities involve counting geodesics paths, not only closed geodesics paths. Alternatively, as in the most classical situation, our consideration corresponds to computing the Hurwitz zeta function instead of the Riemann zeta function. With the same calculations as above, one gets in the finite graph case, (at one point one uses orthogonality of eigenfunctions) the formula

$$
-\log \zeta_{X}(u, x)=\frac{1}{n} \sum_{j=0}^{n-1} f_{j}(x) \bar{f}_{j}\left(x_{0}\right) \log \left(1-\left(q+1-\lambda_{j}\right) u+q u^{2}\right) .
$$

Thus the eigenfunctions come in to determine the more precise count of geodesics. The lead asymptotic as the length goes to infinity behaves the same as for the closed geodesics since the trivial eigenvalue has the constant function as eigenfunction. From an intuitive viewpoint, this observation is clear: For a fixed $x$ and large length $m$ the geodesics do not look much different from a closed geodesic. In symbols, if $m \gg 1$ then $x \approx x_{0}$.

We have the analogous formula for infinite regular graphs, namely that

$$
-\log \zeta_{X}(u, x)=\int \log \left(1-\left(q+1-\lambda_{j}\right) u+q u^{2}\right) d \mu_{x}(\lambda)
$$


Acknowledgments We thank Anton Deitmar, Fabien Friedli and Pierre de la Harpe for reading parts of this paper and alerting us about some inaccuracies.

\section{References}

1. Ahumada, G.: Fonctions périodiques et formule des traces de Selberg sur les arbres. C. R. Acad. Sci. Paris Sér. I Math 305(16), 709-712 (1987)

2. Bass, H.: The Ihara-Selberg zeta function of a tree lattice. Int. J. Math. 3(6), 717-797 (1992)

3. Bednarchak, D.: Heat kernel for regular trees. In harmonic functions on trees and buildings (New York, 1995), volume 206 of Contemp. Math. pp. 111-112. Am. Math. Soc. Providence, RI (1997)

4. Chinta, G., Jorgenson, J., Karlsson, A.: Zeta functions, heat kernels, and spectral asymptotics on degenerating families of discrete tori. Nagoya Math. J. 198, 121-172 (2010)

5. Cowling, M., Meda, S., Setti, A.G.: Estimates for functions of the Laplace operator on homogeneous trees. Trans. Am. Math. Soc. 352(9), 4271-4293 (2000)

6. Clair, B., Mokhtari-Sharghi, S.: Zeta functions of discrete groups acting on trees. J. Algebra 237(2), 591-620 (2001)

7. Chung, F., Yau, S.T.: Coverings, heat kernels and spanning trees. Electron. J. Comb. 6: Research Paper vol. 12, p. 21 (electronic) (1999)

8. Deitmar, A.: Bass-Ihara zeta functions for non-uniform tree lattices. arXiv:1402.4759 [math.NT] (2014)

9. Deitmar, A.: Ihara zeta functions of infinite weighted graphs, arXiv:1402.4945 [math.NT] (2014)

10. Dodziuk, J., Mathai, V.: Kato's inequality and asymptotic spectral properties for discrete magnetic Laplacians. In the ubiquitous heat kernel, volume 398 of Contemp. Math. pp. 69-81. Am. Math. Soc. Providence, RI (2006)

11. Dodziuk, J.: Elliptic operators on infinite graphs. Analysis. geometry and topology of elliptic operators. World Sci. Publ, NJ (2006)

12. Foata, D., Zeilberger, D.: A combinatorial proof of Bass's evaluations of the Ihara-Selberg zeta function for graphs. Trans. Am. Math. Soc. 351(6), 2257-2274 (1999)

13. Guido, D., Isola, T., Lapidus, M.L.: Ihara's zeta function for periodic graphs and its approximation in the amenable case. J. Funct. Anal. 255(6), 1339-1361 (2008)

14. Grigorchuk, R.I., Żuk, A.: The Ihara zeta function of infinite graphs, the KNS spectral measure and integrable maps. In Random walks and geometry. Walter de Gruyter GmbH and Co. KG, Berlin (2004)

15. Hashimoto, K.: Zeta functions of finite graphs and representations of $p$-adic groups. In Automorphic forms and geometry of arithmetic varieties, volume 15 of Adv. Stud. Pure Math. pp. 211-280. Academic Press, Boston (1989)

16. Horton, M.D., Newland, D.B., Terras, A.A.: The contest between the kernels in the Selberg trace formula for the $(q+1)$-regular tree. In the ubiquitous heat kernel, volume 398 of Contemp. Math. pp. 265-293. Am. Math. Soc. Providence, RI (2006)

17. Ihara, Y.: On discrete subgroups of the two by two projective linear group over p-adic fields. J. Math. Soc. Japan 18, 219-235 (1966)

18. Jorgenson, J., Lang, S.: The ubiquitous heat kernel. In mathematics unlimited-2001 and beyond, pp. 655-683. Springer, Berlin (2001)

19. Karlsson, A., Neuhauser, M.: Heat kernels, theta identities, and zeta functions on cyclic groups. In topological and asymptotic aspects of group theory, volume 394 of Contemp. Math. pp. 177-189. Am. Math. Soc. Providence, RI (2006)

20. Kotani, M., Sunada, T.: Zeta functions of finite graphs. J. Math. Sci. Univ. Tokyo 7(1), 7-25 (2000)

21. McKean, H.P.: Selberg's trace formula as applied to a compact Riemann surface. Comm. Pure Appl. Math. 25, 225-246 (1972)

22. Mnëv, P.: Discrete path integral approach to the Selberg trace formula for regular graphs. Commun. Math. Phys. 274(1), 233-241 (2007)

23. Mohar, B., Woess, W.: A survey on spectra of infinite graphs. Bull. Lond. Math. Soc. 21(3), 209-234 (1989)

24. Oberhettinger, F., Badii, L.: Tables of Laplace transforms. Springer, New York (1973)

25. Oren, I., Godel, A., Smilansky, U.: Trace formulae and spectral statistics for discrete Laplacians on regular graphs (I) 4. J. Phys. A Math. Theor. 42(20), 415101 (2009)

26. Pal'tsev, B.V.: On two-sided estimates, uniform with respect to the real argument and index, for modified Bessel functions. Mat. Zametki 65(5), 681-692 (1999) 
27. Serre, J.-P.: Répartition asymptotique des valeurs propres de l'opérateur de Hecke $T_{p}$. J. Am. Math. Soc. 10(1), 75-102 (1997)

28. Serre, J.-P.: Trees. Springer monographs in mathematics. Springer, Berlin. Translated from the French original by John Stillwell, Corrected 2nd printing of the 1980 English translation (2003)

29. Sunada, T.: $L$-functions in geometry and some applications. In curvature and topology of Riemannian manifolds (Katata, 1985), volume 1201 of Lecture Notes in Math. pp. 266-284. Springer, Berlin (1986)

30. Sunada, T.: Fundamental groups and Laplacians [MR0922018 (89d:58128)]. In selected papers on number theory, algebraic geometry, and differential geometry, volume 160 of Am. Math. Soc. Trans. Ser. vol. 2, pp. 19-32. Am. Math. Soc. Providence, RI (1994)

31. Sunada, T.: Discrete geometric analysis. In analysis on graphs and its applications, volume 77 of Proc. Sympos. Pure Math. pp. 51-83. Am. Math. Soc. Providence, RI (2008)

32. Terras, A.: Zeta functions of graphs, volume 128 of Cambridge Studies in advanced mathematics. Cambridge University Press, Cambridge (2011). A stroll through the garden

33. Terras, A.A., Stark, H.M.: Zeta functions of finite graphs and coverings III. Adv. Math. 208(1), 467-489 (2007)

34. Terras, A., Wallace, D.: Selberg's trace formula on the $k$-regular tree and applications. Int. J. Math. Math. Sci. 8, 501-526 (2003) 